\title{
Distribution pattern of carbapenemases and solitary contribution to resistance in clinical strains of Acinetobacter baumannii
}

\author{
Yanpeng Zhang^, Fanglin Ding, Yong Luo, Bing Fan, Zhiyuan Tao, Yanwu Li, Dayong Gu \\ Department of Clinical Laboratory, The First Affiliated Hospital of Shenzhen University, Shenzhen Second People's Hospital, Shenzhen, China \\ Contributions: (I) Conception and design: Y Zhang, D Gu; (II) Administrative support: D Gu, Y Li; (III) Provision of study materials or patients: Z \\ Tao, B Fan; (IV) Collection and assembly of data: Y Zhang, Y Luo; (V) Data analysis and interpretation: Z Tao, F Ding; (VI) Manuscript writing: All \\ authors; (VII) Final approval of manuscript: All authors. \\ Correspondence to: Yanpeng Zhang; Dayong Gu. Department of Clinical Laboratory, The First Affiliated Hospital of Shenzhen University, Shenzhen \\ Second People's Hospital, Shenzhen, China. Email: 61468307@qq.com; wanhood@163.com.
}

\begin{abstract}
Background This study aimed to investigate the distribution pattern of carbapenemases and evaluate their solitary contribution to carbapenem resistance.

Methods: One hundred and twelve isolates of Acinetobacter baumannii (A. baumannii) isolated from the intensive care unit (ICU) of a southern China tertiary hospital were identified, and antimicrobial susceptibility tests (ASTs) of these strains were determined. Common carbapenemases were detected and the distribution pattern of carbapenemases was analyzed. Logistic regression and general linear model analyzed were performed to identify the correlation between antimicrobial susceptibility and carbapenemase genes.

Results: These 112 strains were classified into a carbapenem-resistant $A$. baumannii (CRAB) group (71.7\%) and a carbapenem-susceptible $A$. baumannii (CSAB) group (28.3\%). Carbapenemase genes, including $b l a_{\text {OXA-51-like }}(100.0 \%), b l a_{\text {OXA-23 }}(93.4 \%)$, ISAba1/bla $a_{\text {OXA-51-like }}(27.5 \%), b l a_{\mathrm{NDM}-1}(8.8 \%), b l a_{O X A-24}(2.2 \%)$ and $b l a_{O X A-58}(2.2 \%)$ were detected in CRAB strains, and no $b l a_{\mathrm{SIM}}, b l a_{\mathrm{VIM}}$ and $b l a_{\mathrm{IMP}}$ gene in these 112 isolates. There was a statistically significant difference between CSAB and CRAB group in carrying $b_{a_{\text {OXA-23 }}}(\mathrm{P}<0.001)$ and ISAba1/bla $a_{\text {OXA-51-like }}(\mathrm{P}=0.024)$.

Conclusions: A pattern of $b l a_{\text {OXA-51-like }}(100.0 \%), b l a_{O X A-23}(93.4 \%), b l a_{\mathrm{NDM}-1}(8.8 \%), b l a_{O X A-24}(2.2 \%)$ and $b l a_{\text {OXA-58 }}(2.2 \%)$ was detected in CRAB strains. Bla $a_{\text {OXA-23-like }}$ and ISAba1/bla $a_{\text {OXA-51-like }}$ complex might be more relevant to carbapenem resistance in $A$. baumannii. Harboring $b l a_{\mathrm{OXA}-23 \text {-like }}$ and IS $A b a 1 / b l a_{\mathrm{OXA}-51-\text { like }}$ complex might increase the possibility of resistance 2.16 times [risk ratio (RR): 2.16 ; $95 \%$ confidence interval (CI): $1.04-4.51$ ] and 1.29 times (RR: $1.29 ; 95 \%$ CI: 1.07-1.56), respectively.
\end{abstract}

Keywords: Acinetobacter baumannii (A. baumannii); multidrug resistance; carbapenem; infection; imipenem

Submitted Jun 04, 2021. Accepted for publication Aug 11, 2021.

doi: 10.21037/apm-21-1805

View this article at: https://dx.doi.org/10.21037/apm-21-1805

\section{Introduction}

Acinetobacter baumannii (A. baumannii) is an important nosocomial pathogen. Clinically, it causes ventilatorassociated pneumonia, surgical wound infection, meningitis etc., especially in immune-compromised patients $(1,2)$. As reported in reviews dating back to the 1970s, hospital- acquired pneumonia, especial ventilator-associated pneumonia, was the most common infection caused by A. baumannii (3). According to data of China Bacterial Resistance Monitoring Network (CHINET), A. baumannii accounted for $9.08 \%$ of the total clinical pathogenic isolates and became the third most gram-negative bacteria in the

^ ORCID: 0000-0002-6520-7198. 
clinical bacteria in 2019 in China.

The risk factors of $A$. baumannii infection include invasive operations, surgical treatment and immunosuppression etc. Patients with severe basic diseases in intensive care unit (ICU) commonly need a variety of invasive surgeries and the use of high-dose broad-spectrum antibiotics, which usually leads to internal microecological imbalance and high risk of $A$. baumannii infection. Infection of $A$. baumannii always means a prolonged hospital stay and high mortality. The mortality rate of $A$. baumannii infection is $7.8 \%$ to $23 \%$ in hospital and $10 \%$ to $43 \%$ in ICU (4).

The powerful resistance of $A$. baumannii has been becoming a serious public health problem. Multidrug resistant (MDR), extensively drug resistant and even pan drug resistant strains have been commonly emerging in clinical wards, especially in ICU wards. Carbapenems, such as imipenem and meropenem, either alone or in combination with other category of antibiotics, were once effective therapeutic regimens for $A$. baumannii infections. However, clinical isolates of carbapenem-resistant $A$. baumannii (CRAB) have notably increased in recent years, and data from CHINET showed that resistance rate to imipenem has increased to $73.6 \%$ and $75.1 \%$ in 2019 in China. The CRAB epidemic has become a critical infection problem.

Several resistance mechanisms are involved in $A$. baumannii carbapenem resistance. Generally, production of carbapenemase is thought to be an effective way. Common carbapenemases include OXA-type carbapenemases (5-9) and metallo- $\beta$-lactamases (MBLs) $(5,10,11)$. In addition, up-regulated expression of efflux pump genes (especially AdeABC pump), and down-regulated expression of porin genes may also be associated with carbapenem resistance (12-15).

Surveillance of carbapenemase harboring pattern is necessary for guiding targeted clinical medication in different geographical origin. The drug resistance of $A$. baumannii isolates in different areas is different. There were several reports about carbapenemase harboring pattern in A. baumannii in different areas. However, as far as we know, few studies have focused on the distribution pattern of carbapenem in southern China and the specific contribution of different carbapenems to carbapenem antibiotic resistance. This study aims to investigate the distribution pattern of carbapenemases to evaluate (using statistical methods) their solitary contribution to carbapenem resistance.

We present the following article in accordance with the MDAR reporting checklist (available at https://dx.doi. org/10.21037/apm-21-1805).

\section{Methods}

\section{Isolation and identification of strains}

In total, 125 non-repetitive strains of the Acinetobacter calcoaceticus-Acinetobacter baumannii complex were collected from the patients of ICU of a tertiary hospital, Shenzhen, China, from July 2018 to June 2019. The specimen source included sputum, nasal secretion samples, etc.

The collected strains were inoculated on McConkey plate, and the single colony overnight was used for identification. According to the manufacturer's guidelines, 125 isolates were identified as Acinetobacter calcoaceticusAcinetobacter baumannii complex using the AutoMicrobic system Gram-Negative Identification Card on microbiology analyzer VITEK 2 system (Biomerieux, France). 112 in 125 isolates were further confirmed as $A$. baumannii by $r p o B$ gene sequence analysis assays according to previous established polymerase chain reaction (PCR) conditions.

This study was approved by the Ethics Committee of the First Affiliated Hospital of Shenzhen University (ID: 20200511007). This was a retrospective study obtaining data from hospital's information system. The results of this study may benefit patients with $A$. baumannii infection and may lead to better therapeutic outcomes. The requirement for informed consent of patients in this study was waived. All procedures performed in this study involving human participants were in accordance with the Declaration of Helsinki (as revised in 2013).

\section{Antimicrobial susceptibility test (AST)}

AST of these isolates was determined by an automated broth microdilution method (gram-negative susceptibility cards) using the VITEK 2 system. Susceptibility interpretation was based on clinical breakpoint from the Clinical and Laboratory Standards Institute (CLSI 2019, $29^{\text {th }}$ edition; DocumentM100). These antimicrobials included cefazolin, cefotetan, ampicillin + sulbactam, piperacillin + tazobactam, ceftriaxone, ceftazidime, cefepime, ciprofloxacin, levofloxacin, tobramycin, gentamycin, nitrofurantoin. Escherichia coli ATCC 25922 and Pseudomonas aeruginosa ATCC 27853 were used as quality control.

According to AST result, 112 strains were divided into carbapenem-susceptible $A$. baumannii (CSAB) group and 
Table 1 Primer sequences and amplicon size

\begin{tabular}{|c|c|}
\hline Target genes & Primer sequence \\
\hline \multirow[t]{2}{*}{ rрoв } & F: GAGTCTAATGGCGGTGGTTC \\
\hline & R: ATTGCTTCATCTGCTGGTTG \\
\hline \multicolumn{2}{|c|}{ OXA type carbapenemases } \\
\hline \multirow[t]{2}{*}{ bla $_{\text {OXA-23-like }}$} & F: GAATATGTGCCAGCCTCTAC \\
\hline & R: GCATTACCGAAACCAATACG \\
\hline \multirow[t]{2}{*}{ bla ${ }_{O X A-24-l i k e}$} & F: TGGGTGGAGCAAGCTAATGG \\
\hline & R: ACGAATAGAACCAGACATTCCTTCT \\
\hline \multirow[t]{2}{*}{$b l a_{O X A-51-l i k e}$} & F: TAATGCTTTGATCGGCCTTG \\
\hline & R: TGGATTGCACTTCATCTTGG \\
\hline \multirow[t]{2}{*}{$b a_{O X A-58-l i k e}$} & F: GACAATTACACCTATACAAGAAG \\
\hline & R: AAACCCACATACCAACCC \\
\hline \multirow[t]{2}{*}{ ISAba1/bla ${ }_{\text {OXA-51 }}$} & F: CACGAATGCAGAAGTTG \\
\hline & R: CTTCTGTGGTGGTTGGC \\
\hline
\end{tabular}

MBLs type carbapenemases

\begin{tabular}{|c|c|}
\hline \multirow[t]{2}{*}{$b / a_{I M P}$} & F: GGAATAGAGTGGCTTAAYTC \\
\hline & R: TCGGTTTAAYAAAACAACCACC \\
\hline \multirow[t]{2}{*}{$b a_{N D M-1}$} & F: GGTTTGGCGATCTGGTTTTC \\
\hline & R: CGGAATGGCTCATCACGATC \\
\hline \multirow[t]{2}{*}{$b / a_{N D M-1}$} & F: GAGTATTCAACATTTCCGTGTC \\
\hline & R: TAATCAGTGAGGCACCTATCTC \\
\hline \multirow[t]{2}{*}{$b l a_{S I M}$} & F: TACAAGGGATTCGGCATCG \\
\hline & R: TAATGGCCTGTTCCCATGTG \\
\hline \multirow[t]{2}{*}{$b l a_{V I M}$} & F: GATGGTGTITGGTCGCATA \\
\hline & R: CGAATGCGCAGCACCAG \\
\hline \multicolumn{2}{|c|}{ AdeABC efflux pump } \\
\hline \multirow[t]{2}{*}{ ade $B$} & F: GCAGAGCGTACTCGGAATGT \\
\hline & R: CCACTGAAACCCCATCCCAA \\
\hline
\end{tabular}

MBLs, metallo- $\beta$-lactamases.

CRAB group.

\section{Detection of carbapenemase gene}

Common carbapenemase genes of $A$. baumannii, including $b l a_{\text {OXA-51-like }}, b l a_{\text {OXA-23-like }}, b l a_{\text {OXA-24-like, }}, b l a_{\text {OXA-58-like }}, b l a_{\text {SIM }}$, $b l a_{\mathrm{VIM}}, b l a_{\mathrm{IMP}}, b l a_{\mathrm{NDM}-1}$ and ISAba1/bla $a_{\mathrm{OXA}-51 \text {-like }}$ were detected according to previously established PCR conditions $(13,16,17)$. All PCR primers used in this study were listed in
Table 1. Four A. baumannii strains, ab606, ab608, ab609, and SZE, were used as positive quality control strains.

\section{Statistical analysis}

Stata/SE 15.1 (StataCorp LLC, TX, USA) was used for data entry and statistical analysis. All tests were two-tailed, and a $P$ value $\leq 0.05$ was considered statistically significant. Sample demographic and disease characteristic were compared using Pearson's chi-square test. AST and harboring of resistance genes were compared between the CSAB and CRAB group using Fisher-Exact test. Logistic Regression was used to assess the relations between harboring of resistance gene and antimicrobial susceptibility result. General regression model was used to make multivariate regression analysis of resistance genes and antimicrobial susceptibility results to calculate the risk ratio (RR).

\section{Results}

\section{Clinical characteristics of $A$. baumannii strains}

As summarized in Table 2, a total of 112 patients were included in this study, including 70 males $(62.5 \%)$ and 42 females $(37.5 \%)$. Seventy-eight $(69.6 \%)$ patients were over 60 years old and $32(28.6 \%)$ were between 16 to 59 years old. The causes of ICU admission were respiratory distress in 37 cases $(33.0 \%)$, poor general condition in 27 cases (24.1\%), trauma in 29 cases (25.9\%), and other causes in 19 cases $(17.0 \%)$.

\section{$A S T$}

AST results of $112 A$. baumannii strains are summarized in Figure 1. Of the 112 isolates, 21 strains (18.8\%) were in $\mathrm{CSAB}$ group and 91 strains (81.2\%) in CRAB group. CRAB strains showed almost $100 \%$ resistant to cephalosporins (ceftriaxone, ceftazidime, cefepime), over $70 \%$ resistant to quinolones (ciprofloxacin, levofloxacin) and aminoglycosides (tobramycin, gentamicin). In this study, there was evidence that the combination preparation such as sulbactam and cefoperazone was a reasonable choice for the treatment of CRAB infection.

\section{Distribution of carbapenemase}

As shown in Table 3, bla $a_{\mathrm{SIM}}, b l a_{\mathrm{VIM}}$ and $b l a_{\mathrm{IMP}}$ genes were not detected in any of the 112 isolates, and $b l a_{\text {OXA-51-like }}$ 
gene was found in all $112(100.0 \%)$ isolates. The positive rate of bla OXA-23-like $_{\text {was }} 93.4 \%$ in CRAB group, and $19 \%$ in CSAB group. Compared with CSAB, CRAB strains showed a statistically significant increasing distribution of $b l a_{\text {OXA-23-like }}(\mathrm{P}<0.001)$ and ISAba1/bla $a_{\text {OXA-51-like }}(\mathrm{P}=0.024)$, respectively. $B l a_{\mathrm{OXA}-24-\text { like }}$ and $b l a_{\mathrm{OXA}-58 \text {-like }}$ were found in $2.2 \%$

Table 2 Sample demographic and disease characteristic

\begin{tabular}{lcc}
\hline Characteristics & Number & $\%$ \\
\hline Age & 2 & 1.8 \\
$\leq 15$ & 32 & 28.6 \\
$16-59$ & 78 & 69.6 \\
$\geq 60$ & & \\
Gender & 42 & 37.5 \\
Female & 70 & 62.5 \\
Male & & \\
Reason of ICU admission & 37 & 33.0 \\
Respiratory distress & 27 & 24.1 \\
Poor general condition & 29 & 25.9 \\
Trauma & 19 & 17.0 \\
Others & & \\
\hline
\end{tabular}

ICU, intensive care unit. strains, which belonged to CRAB group, and $b l a_{N D M-1}$ gene was detected in $8.8 \%$ strains. No $b l a_{\mathrm{SIM}}, b l a_{\mathrm{VIM}}$ and $b l a_{\mathrm{IMP}}$ genes were detected in these isolates.

\section{Univariate logistic analysis and multivariate regression analysis for carbapenem resistance}

As presented in Table 4, bla $a_{\text {OXA-23-like, }}$, $l a_{\text {OXA-24-like, IS } A b a 1 /}$ $b l a_{\mathrm{OXA}-51 \text {-like }}$, and $a d e B$ were included to perform the univariate logistic and multivariate regression analyses. Two variables, $b l a_{\text {OXA-24-like }}$ and $b l a_{\text {OXA-58-like, }}$ were dropped automatically by Stata/SE statistical software.

Based on the result of multivariate regression analysis, carrying $b l a_{\mathrm{OXA}-23-\text { like }}$ and IS $A b a 1 / b l a_{\mathrm{OXA}-51 \text {-like }}$ might increase the possibility of carbapenem resistance by 2.16 times [adjusted RR $=2.16$; 95\% confidence interval (CI): 1.04 4.51] and 1.29 times (adjusted RR $=1.29$; $95 \%$ CI: $1.07-$ $1.56)$, respectively.

\section{Discussion}

A. baumannii is an important nosocomial pathogen, which can cause ventilator-associated pneumonia, surgical wound infection, meningitis etc. A. baumannii can enter the bloodstream and cause bacteremia, which may further develop into sepsis. A. baumannii infection leads to a high clinical mortality. In this decade, an increasing number of

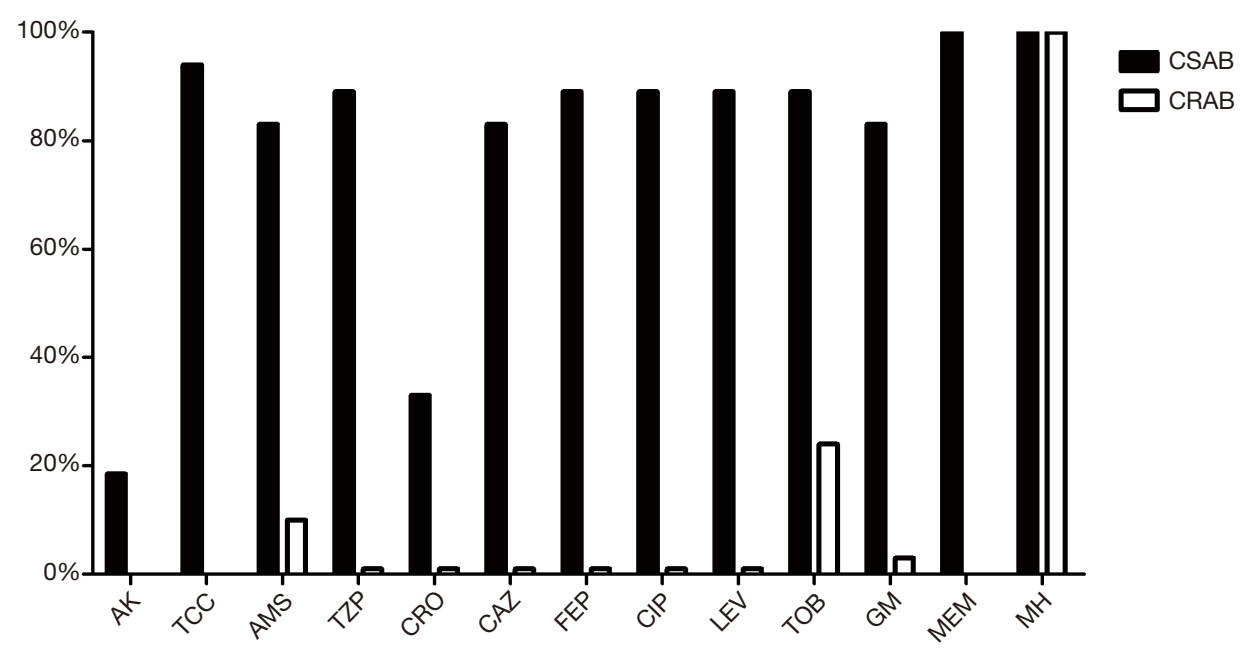

Figure 1 Percentage of antibiotic sensitivity of CSAB and CRAB strains. CSAB, carbapenem-susceptible A. baumannii; CRAB, carbapenemresistant A. baumannii; A. baumannii, Acinetobacter baumannii; AK, amikacin; TCC, ticarcillin-clavulanic acid; AMS, ampicillin-sulbactam; TZP, piperacillin-tazobactam; CRO, ceftriaxone; CAZ, ceftazidime; FEP, cefepime; CIP, ciprofloxacin; LEV, levofloxacin; TOB, tobramycin; GM, gentamicin; MEM, meropenem; MH, minocycline. 
Table 3 Distribution pattern of carbapenemases

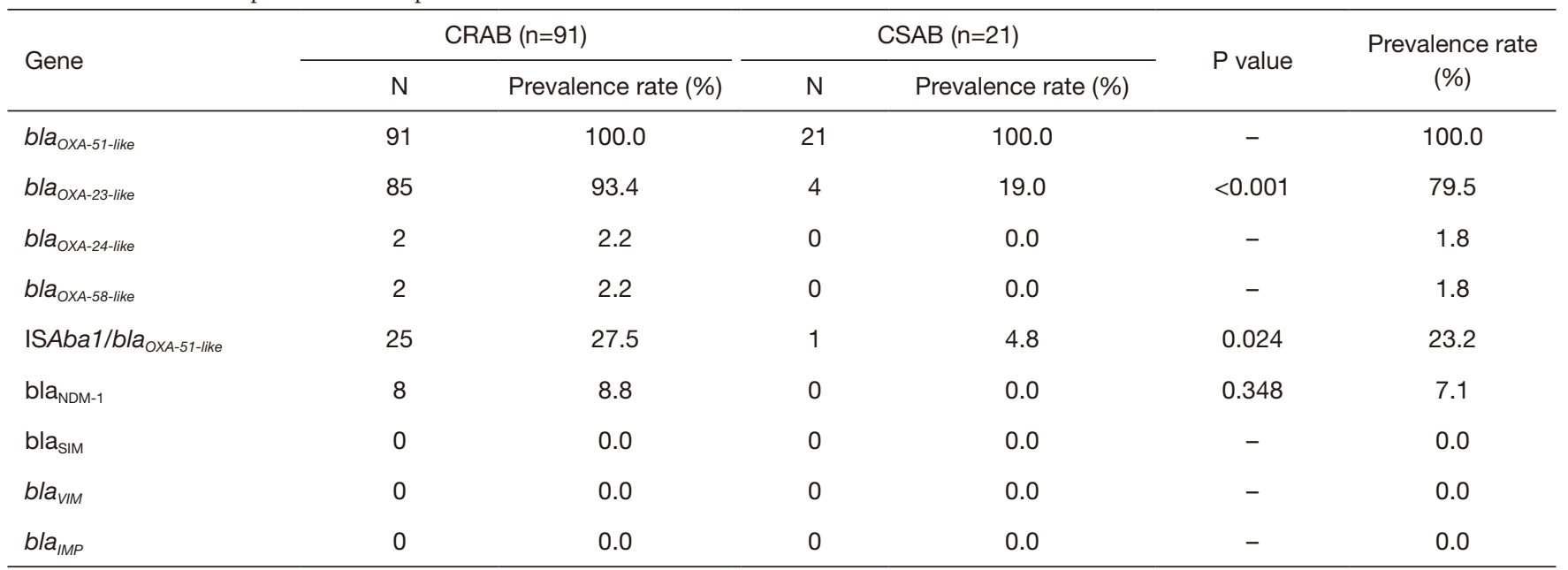

CRAB strains. CSAB, carbapenem-susceptible A. baumannii; CRAB, carbapenem-resistant A. baumannii; A. baumannii, Acinetobacter baumannii.

Table 4 Univariate logistic and multivariate regression analyses for carbapenem resistance

\begin{tabular}{|c|c|c|c|c|c|c|}
\hline \multirow{3}{*}{ Variables } & \multicolumn{6}{|c|}{ Carbapenem resistance } \\
\hline & \multicolumn{3}{|c|}{ Univariate logistic analysis } & \multicolumn{3}{|c|}{ Multivariate regression analysis } \\
\hline & OR & $P$ value & $95 \% \mathrm{Cl}$ & $\mathrm{RR}$ & $P$ value & $95 \% \mathrm{Cl}$ \\
\hline$b l a_{\text {OXA-23-like }}$ & 7.62 & $<0.01$ & $2.28-25.48$ & 2.16 & 0.03 & $1.04-4.51$ \\
\hline ISAba1/bla OXA-51-like & 12.49 & 0.01 & $1.58-98.39$ & 1.29 & 0.01 & $1.07-1.56$ \\
\hline
\end{tabular}

$\mathrm{OR}$, odds ratio; $\mathrm{Cl}$, confidence interval; $\mathrm{RR}$, risk ratio.

pan-drug resistant isolates have been reported globally. This indicated that $A$. baumannii strains with powerful resistance ability have been becoming a serious problem for clinical infection control. As reported, relevant mechanisms to carbapenem resistance include enzymehydrolyzing, overproduction of efflux pump, changing of outer membrane and biofilm formation (16-18). Monitoring molecular characteristic is necessary for taking effective measures to control clinical infection. In view of the complex mechanism of drug resistance, it is difficult to evaluate the contribution of different carbapenem enzymes. In this study, we found carrying $b l a_{\text {OXA-23-like }}$ and ISAba1/ $b l a_{\text {OXA-51-like }}$ might increase the possibility of carbapenem resistance by 2.16 times (adjusted $\mathrm{RR}=2.16$; $95 \% \mathrm{CI}$ : 1.04-4.51) and 1.29 times (adjusted $\mathrm{RR}=1.29$; $95 \% \mathrm{CI}$ : 1.07-1.56), respectively. Compared with CSAB strains, CRAB strains showed a statistically significant increasing distribution of $b l a_{\mathrm{OXA}-23-\text { like }}(\mathrm{P}<0.001)$ and ISAba1/bla $a_{\text {OXA-51-like }}$ $(\mathrm{P}=0.024)$.
Elderly patients with underlying diseases, highly selective antibiotics and mechanical ventilation, are an important population for nosocomial infection of $A$ baumannii. In this study, we found $69.9 \%$ of $A$. baumannii infected patients were $\geq 60$ years old, and most of them had fundamental illness. We also found that $62.5 \%$ of infected patients were male and had an extended period of hospitalization, which was consistent with the report of Wisplinghoff et al. (19).

Carbapenem was once regarded as main antibacterial drug for A. baumannii infection (20), but the susceptibility has decreased in the recent years. In this study, $81.3 \%$ of A. baumannii isolates were CRAB, which was consistent with the report in Thailand (21). The resistance rate of ceftazidime and other third generation cephalosporins was over $75 \%$, and that of solitary quinolones or aminoglycosides was basically over $60 \%$.

Clinically, a combination of different category of antibiotic is common option for CRAB infection (22). The combination of cefoperazone and sulbactam showed a good 
performance in treating infection of CRAB. Tigecycline and polymyxin are other drugs commonly used in $\mathrm{CRAB}$ infection. Nevertheless, the effect of tigecycline remains controversial. Some research concluded that tigecycline was be effective, but other clinical research regarded tigecycline might be associated with higher in-hospital mortality and lower microbial eradication rate $(23,24)$.

Strong resistance of $A$. baumannii is due to its genomic plasticity. Under selective pressure of antibiotics, $A$. baumannii can acquire resistance through two ways: (I) integration of resistance determinants by horizontal transfer of mobile elements (25); and (II) regulation intrinsic resistance mechanisms, including up-regulation of some intrinsic hydrolases and efflux pumps, down-regulation of membrane permeability, formation of biofilm (1,2,26-29).

Carbapenemases are $\beta$-lactamases that confer resistance to carbapenem, penicillin and cephalosporin. According to the Ambler classification system, common carbapenemases in A. baumannii include B class (MBLs) and D class (OXA-type carbapenemases). The former includes $b l a_{\mathrm{SIM}}$, $b l a_{\mathrm{VIM}}, b l a_{\mathrm{IMP}}$, and $b l a_{\mathrm{NDM}}$. The latter includes $b l a_{\mathrm{OXA}-51 \text {-like, }}$, $b l a_{\text {OXA-23-like, }} b l a_{\text {OXA-24-like, }}$ and $b l a_{\text {OXA-58-like. }} . B l a_{\text {OXA-23-like }}$ gene cluster (encoding OXA-23, OXA-27 and OXA-49) has been shown to contribute to carbapenem resistance. $B l a_{\text {OXA-24-like }}$ is another OXA type carbapenemase gene cluster, which encodes OXA-24, -25, -26, and -40.

The strains of different geographical origin had different carbapenemase harboring patterns. Koirala et al. found $b l a_{\mathrm{OXA}-23}(52 \%)$ and $b l a_{\mathrm{OXA}-40}(28 \%)$ were the most frequent genes among carbapenem non-susceptible isolates in Illinois (USA) (30). Indian scholar observed $97.7 \%$ of CRAB harboring $b l a_{\text {OXA-23-like }}$ gene, $b l a_{\mathrm{NDM}-1}(29.1 \%)$ and $b l a_{\text {OXA-58-like }}$ (3.5\%) in India (31). And in Thailand, a pattern of $b l a_{\mathrm{OXA}-23}$ $(82.6 \%), b l a_{\mathrm{NDM}-1}(9.1 \%), b l a_{\mathrm{OXA}-24}(0.3 \%)$ and $b l a_{\mathrm{OXA}-58}$ $(6.5 \%)$ was reported (21). In this study, we found a pattern of $b l a_{\text {OXA-51-like }}(100.0 \%), b l a_{O X A-23}(93.4 \%), b l a_{\mathrm{NDM}-1}(8.8 \%)$, $b l a_{O X A-24}(2.2 \%)$ and $b l a_{O X A-58}(2.2 \%)$ in CRAB strains. And the harboring of $b l a_{\text {OXA-23-like }}$ increased the possibility of $A$. baumannii carbapenem resistance by 2.16 times (adjusted $\mathrm{RR}=2.16$; 95\% CI: 1.04-4.51) in these strains.

The chromosomal gene $b l a_{\text {OXA-51-like }}$ is intrinsic in A. baumannii. Harboring bla $a_{\mathrm{OXA}-51-\mathrm{like}}$ gene alone can hardly cause carbapenem resistance. IS $A b a 1 / b l a_{\text {OXA-51-like }}$ complex is $b l a_{\text {OXA-51-like }}$ with an additional genetic element IS $A b a 1$ upstream insertion. This structure can result in overexpression of OXA-51-like enzyme and confers resistance to carbapenems. Notably, in this study we detected $27.5 \%$ isolates carrying IS $A b a 1 / b l a_{\text {OXA-51-like, which }}$ belonged to CRAB group, and no IS $A b a 1 / b l a_{\text {OXA-51-like }}$ complex was found in the CSAB strains. Also, the presence of ISAba1/bla $a_{\text {OXA-51-like }}$ complex was significantly associated with resistance of carbapenem (adjusted RR $=1.29 ; 95 \%$ CI: 1.07-1.56), which indicated that IS $A b a 1 / b l a_{\text {OXA-51-like }}$ could play an important role in carbapenem resistance.

Furthermore, over-expression of AdeABC efflux pump was associated with carbapenem resistance. In this study, we found no significant difference in carrying $a d e B$ gene between $C R A B$ group and $C S A B$ group $(\mathrm{P}=0.51>0.05)$. This result may be because our study only involved the detection of pump gene, but did not discuss the expression of this gene. We will conduct further research on the contribution of overproduction of different efflux pumps in the future.

A. baumannii drug resistance has been increasing in these years, which has brought severe challenge to clinical treatment. To reverse this situation, on the one hand, the use of antibiotics outside the indications should be strictly controlled and antibiotics should be selected according to the results of AST. On the other hand, we should strengthen the surveillance of distribution pattern of drug resistance genes to prevent or delay the emergence of drugresistant strains.

\section{Limitations}

This study had several limitations. Firstly, the source of the strains was only from one hospital, and collection was conducted over a short period of time. Secondly, the regulation of efflux pump gene expression was not discussed in this study. This study only focused on the common drug resistance mechanism of $A$. baumannii, and did not involve other mechanisms, such as the low production of outer membrane protein affecting membrane permeability and the over production of efflux pump.

\section{Conclusions}

A pattern of bla $a_{\text {OXA-51-like }}(100.0 \%), b l a_{O X A-23}(93.4 \%)$, $b l a_{\mathrm{NDM}-1}(8.8 \%), b l a_{O X A-24}(2.2 \%)$ and $b l a_{O X A-58}(2.2 \%)$ was detected in CRAB strains, and bla $a_{\text {OXA-23-like }}$ and ISAba1/ $b l a_{\text {OXA-51-like }}$ complex might be more relevant to carbapenem resistance in A. baumannii. We also evaluated their solitary contribution to carbapenem resistance by statistical methods. These findings could help to develop effective regimens and prevention strategies for controlling $A$. 
baumannii infections.

\section{Acknowledgments}

The authors thank the engineers of the medical information system.

Funding: This project was financially supported by a grant from the Shenzhen Science and Technology Foundation (No. JCYJ20190806164011195).

\section{Footnote}

Reporting Checklist: The authors have completed the MDAR reporting checklist. Available at https://dx.doi. org/10.21037/apm-21-1805

Data Sharing Statement: Available at https://dx.doi. org/10.21037/apm-21-1805

Conflicts of Interest: All authors have completed the ICMJE uniform disclosure form (available at https://dx.doi. org/10.21037/apm-21-1805). The authors have no conflicts of interest to declare.

Ethical Statement: The authors are accountable for all aspects of the work in ensuring that questions related to the accuracy or integrity of any part of the work are appropriately investigated and resolved. This study was approved by the Ethics Committee of the First Affiliated Hospital of Shenzhen University (ID: 20200511007). This was a retrospective study obtaining data from hospital's information system. The requirement for informed consent of patients in this study was waived. All procedures performed in this study involving human participants were in accordance with the Declaration of Helsinki (as revised in 2013).

Open Access Statement: This is an Open Access article distributed in accordance with the Creative Commons Attribution-NonCommercial-NoDerivs 4.0 International License (CC BY-NC-ND 4.0), which permits the noncommercial replication and distribution of the article with the strict proviso that no changes or edits are made and the original work is properly cited (including links to both the formal publication through the relevant DOI and the license). See: https://creativecommons.org/licenses/by-nc-nd/4.0/.

\section{References}

1. Antunes LC, Visca P, Towner KJ, et al. Acinetobacter baumannii: evolution of a global pathogen. Pathog Dis 2014;71:292-301.

2. Harding CM, Hennon SW, Feldman MF. Uncovering the mechanisms of Acinetobacter baumannii virulence. Nat Rev Microbiol 2018;16:91-102.

3. Roca I, Espinal P, Vila-Farrés X, et al. The Acinetobacter baumannii oxymoron: commensal hospital dweller turned pan-drug-resistant menace. Front Microbiol 2012;3:148.

4. Falagas ME, Bliziotis IA, Siempos II, et al. Attributable mortality of Acinetobacter baumannii infections in critically ill patients: a systematic review of matched cohort and case-control studies. Crit Care 2006;10:R48.

5. Rodríguez CH, Nastro M, Famiglietti A. Carbapenemases in Acinetobacter baumannii. Review of their dissemination in Latin America. Rev Argent Microbiol 2018;50:327-33.

6. Mendes RE, Bell JM, Turnidge JD, et al. Emergence and widespread dissemination of OXA-23, -24/40 and -58 carbapenemases among Acinetobacter spp. in Asia-Pacific nations: report from the SENTRY Surveillance Program. J Antimicrob Chemother 2009;63:55-9.

7. Karampatakis T, Tsergouli K, Politi L, et al. Polyclonal predominance of concurrently producing OXA-23 and OXA-58 carbapenem-resistant Acinetobacter baumannii strains in a pediatric intensive care unit. Mol Biol Rep 2019;46:3497-500.

8. Opazo A, Domínguez M, Bello H, et al. OXA-type carbapenemases in Acinetobacter baumannii in South America. J Infect Dev Ctries 2012;6:311-6.

9. Nigro S, Hall RM, et al. Distribution of the blaOXA-23containing transposons Tn2006 and Tn2008 in Australian carbapenem-resistant Acinetobacter baumannii isolates. J Antimicrob Chemother 2015;70:2409-11.

10. Routray A, Lavanya P, Soniya R, et al. Multiplex PCR for genes encoding prevalent OXA and NDM1 carbapenemases in Acinetobacter. J Pharm Res 2013;7:324-6.

11. Maamar E, Alonso CA, Ferjani S, et al. NDM-1- and OXA-23-producing Acinetobacter baumannii isolated from intensive care unit patients in Tunisia. Int J Antimicrob Agents 2018;52:910-5.

12. Yang YS, Chen HY, Hsu WJ, et al. Overexpression of AdeABC efflux pump associated with tigecycline resistance in clinical Acinetobacter nosocomialis isolates. Clin Microbiol Infect 2019;25:512.e1-6. 
13. Zhang Y, Li Z, He X, et al. Overproduction of efflux pumps caused reduced susceptibility to carbapenem under consecutive imipenem-selected stress in Acinetobacter baumannii. Infect Drug Resist 2018;11:457-67.

14. Nasr P. Genetics, epidemiology, and clinical manifestations of multidrug-resistant Acinetobacter baumannii. J Hosp Infect 2020;104:4-11.

15. El-Shazly S, Dashti A, Vali L, et al. Molecular epidemiology and characterization of multiple drugresistant (MDR) clinical isolates of Acinetobacter baumannii. Int J Infect Dis 2015;41:42-9.

16. Mentasti M, Prime K, Sands K, et al. Rapid detection of OXA-23-like, OXA-24-like, and OXA-58-like carbapenemases from Acinetobacter species by real-time PCR. J Hosp Infect 2020;105:741-6.

17. Turton JF, Ward ME, Woodford N, et al. The role of ISAba1 in expression of OXA carbapenemase genes in Acinetobacter baumannii. FEMS Microbiol Lett 2006;258:72-7.

18. Abdi SN, Ghotaslou R, Ganbarov K, et al. Acinetobacter baumannii Efflux Pumps and Antibiotic Resistance. Infect Drug Resist 2020;13:423-34.

19. Wisplinghoff H, Perbix $W$, Seifert $H$, et al. Risk factors for nosocomial bloodstream infections due to Acinetobacter baumannii: a case-control study of adult burn patients. Clin Infect Dis 1999;28:59-66.

20. Doi Y. Treatment options for carbapenem-resistant gram-negative bacterial infections. Clin Infect Dis 2019;69:S565-75.

21. Leungtongkam $\mathrm{U}$, Thummeepak R, Wongprachan S, et al. Dissemination of blaOXA-23, blaOXA-24, blaOXA-58, and blaNDM-1 Genes of Acinetobacter baumannii Isolates from Four Tertiary Hospitals in Thailand. Microb Drug Resist 2018;24:55-62.

22. Viehman JA, Nguyen MH, Doi Y, et al. Treatment options for carbapenem-resistant and extensively drugresistant Acinetobacter baumannii infections. Drugs 2014;74:1315-33.

Cite this article as: Zhang Y, Ding F, Luo Y, Fan B, Tao Z, Li Y, Gu D. Distribution pattern of carbapenemases and solitary contribution to resistance in clinical strains of Acinetobacter baumannii. Ann Palliat Med 2021;10(8):9184-9191. doi: 10.21037/ apm-21-1805
23. Ni W, Han Y, Zhao J, et al. Tigecycline treatment experience against multidrug-resistant Acinetobacter baumannii infections: a systematic review and metaanalysis. Int J Antimicrob Agents 2016;47:107-16.

24. Mei H, Yang T, Wang J, et al. Efficacy and safety of tigecycline in treatment of pneumonia caused by MDR Acinetobacter baumannii: a systematic review and metaanalysis. J Antimicrob Chemother 2019;74:3423-31.

25. Fournier PE, Vallenet D, Barbe V, et al. Comparative genomics of multidrug resistance in Acinetobacter baumannii. PLoS Genet 2006;2:e7.

26. Siroy A, Molle V, Lemaître-Guillier C, et al. Channel formation by $\mathrm{CarO}$, the carbapenem resistance-associated outer membrane protein of Acinetobacter baumannii. Antimicrob Agents Chemother 2005;49:4876-83.

27. del Mar Tomás M, Beceiro A, Pérez A, et al. Cloning and functional analysis of the gene encoding the 33- to 36-kilodalton outer membrane protein associated with carbapenem resistance in Acinetobacter baumannii. Antimicrob Agents Chemother 2005;49:5172-5.

28. Rumbo C, Gato E, López M, et al. Contribution of efflux pumps, porins, and $\beta$-lactamases to multidrug resistance in clinical isolates of Acinetobacter baumannii. Antimicrob Agents Chemother 2013;57:5247-57.

29. Bratu S, Landman D, Martin DA, et al. Correlation of antimicrobial resistance with beta-lactamases, the OmpA-like porin, and efflux pumps in clinical isolates of Acinetobacter baumannii endemic to New York City. Antimicrob Agents Chemother 2008;52:2999-3005.

30. Koirala J, Tyagi I, Guntupalli L, et al. OXA-23 and OXA-40 producing carbapenem-resistant Acinetobacter baumannii in Central Illinois. Diagn Microbiol Infect Dis 2020;97:114999.

31. Kumar S, Patil PP, Singhal L, et al. Molecular epidemiology of carbapenem-resistant Acinetobacter baumannii isolates reveals the emergence of blaOXA-23 and blaNDM-1 encoding international clones in India. Infect Genet Evol 2019;75:103986. 